\section{Controlling the dynamics of spontaneous emission from quantum dots by photonic crystals}

Peter Lodahl $^{1}$, A. Floris van Driel ${ }^{2}$, Ivan S. Nikolaev ${ }^{1}$, Arie Irman ${ }^{1}$, Karin Overgaag ${ }^{1}$, Daniël Vanmaekelbergh ${ }^{2} \&$ Willem L. Vos ${ }^{1}$

${ }^{1}$ Complex Photonic Systems (COPS), Department of Science and Technology and

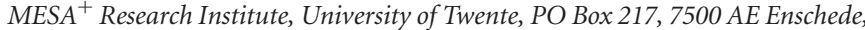
The Netherlands

${ }^{2}$ Debye Institute, Condensed Matter and Interfaces, Utrecht University, PO Box 80.000, 3508 TA Utrecht, The Netherlands

Control of spontaneously emitted light lies at the heart of quantum optics. It is essential for diverse applications ranging from miniature lasers and light-emitting diodes ${ }^{1-5}$, to singlephoton sources for quantum information ${ }^{6-8}$, and to solar energy harvesting ${ }^{9}$. To explore such new quantum optics applications, a suitably tailored dielectric environment is required in which the vacuum fluctuations that control spontaneous emission can be manipulated ${ }^{10,11}$. Photonic crystals provide such an environment: they strongly modify the vacuum fluctuations, causing the decay of emitted light to be accelerated or slowed down ${ }^{12,13}$, to reveal unusual statistics ${ }^{14}$, or to be completely inhibited in the ideal case of a photonic bandgap ${ }^{1,15}$. Here we study spontaneous emission from semiconductor quantum dots embedded in inverse opal photonic crystals ${ }^{16}$. We show that the spectral distribution and time-dependent decay of light emitted from excitons confined in the quantum dots are controlled by the host photonic crystal. Modified emission is observed over large frequency bandwidths of $10 \%$, orders of magnitude larger than reported for resonant optical microcavities ${ }^{17}$. Both inhibited and enhanced decay rates are observed depending on the optical emission frequency, and they are controlled by the crystals' lattice parameter. Our experimental results provide a basis for all-solid-state dynamic control of optical quantum systems ${ }^{18}$.

The characteristics of spontaneously emitted light depend strongly on the environment of the emitter; both the total rate of emission and the direction-dependent spectra are affected. In the Wigner-Weisskopf approximation ${ }^{10}$, the radiative decay rate is described by Fermi's 'golden rule ${ }^{11}$ that contains the local density of electromagnetic states ${ }^{19,20}$ (LDOS). The LDOS counts the available number of electromagnetic modes in which photons can be emitted at the specific location of the emitter, and can be interpreted as the density of vacuum fluctuations. The LDOS and hence the emission rate depend sensitively on the emission frequency $\omega$ and on the position of the emitter, but not on the emission direction. Here we report on the frequency-dependent emission rate of quantum dots under the influence of modified vacuum fluctuations in photonic crystals.

In photonic crystals (Fig. 1a), emission of light is modified because the refractive index varies spatially on length scales of the order of the wavelength. Hence, light interference by Bragg diffraction causes characteristic stopbands leading to direction-dependent spectra. The emission rate is modified if Bragg stop bands exclude a substantial solid angle of the crystal. To confirm the influence of Bragg diffraction, we have performed angle-resolved measurements of the emission spectra of light sources embedded in titania inverse opals (see Methods). As light sources, we use colloidal CdSe quantum dots that are highly efficient, narrow linewidth, sizedependent emitters that effectively resemble two-level systems. Figure $1 \mathrm{~b}$ shows the emission spectra for a sample with lattice parameter $a=420 \mathrm{~nm}$ for different detection angles $\alpha$ relative to the (111) planes. An unusual angular dependence is observed because the emitted intensity is strongly attenuated at $\alpha=0^{\circ}$, increases at $\alpha=20^{\circ}$ and $\alpha=50^{\circ}$, and decreases again at $\alpha=60^{\circ}$. This behaviour is caused by a photonic stopband centred at $\alpha=0^{\circ}$ that is instantly recognized when the emission spectra are referenced to spectra measured at $\alpha=60^{\circ}$. The latter are suitable references because here the stopbands are shifted far outside the emission spectrum of the quantum dots.

Figure 1c displays the angular dependence of the relative intensity for samples with three different lattice parameters. For a reference
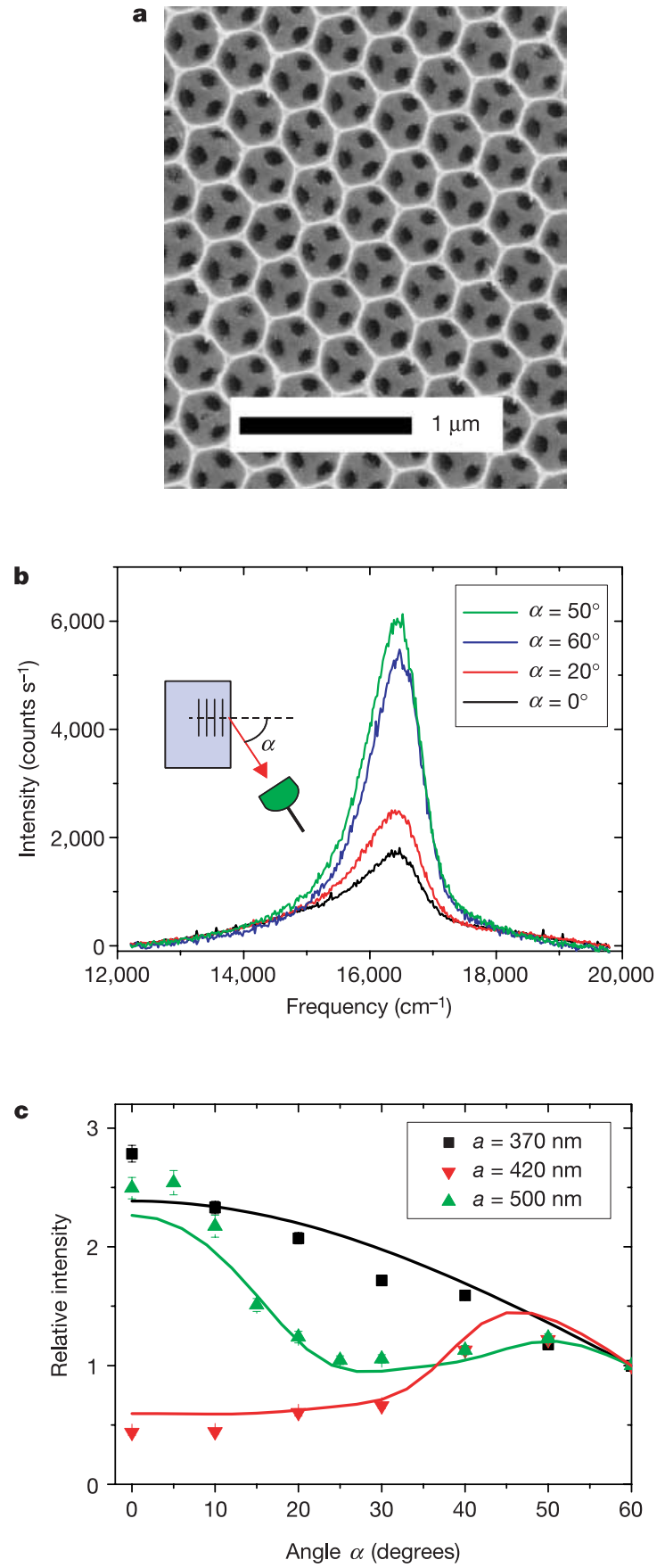

Figure 1 Angle-resolved spectral measurements. a, Scanning electron microscope image of the (111) face of a titania inverse opal with lattice parameter $a=460 \mathrm{~nm}$. b. Recorded quantum dot emission spectra from an inverse opal with lattice parameter $a=420 \mathrm{~nm}$ for different detection angles $\alpha$ relative to the (111) lattice planes.

c, Measured (points) and calculated (curves) angle-dependent intensities at fixed frequency $\omega=15,870 \mathrm{~cm}^{-1}$ for three samples with different lattice parameters. The intensities are divided by the intensity measured at $\alpha=60^{\circ}$, where no stopbands appear. 
sample with a small lattice parameter $(a=370 \mathrm{~nm})$, the stopgaps appear at higher frequencies than the emission spectrum of the quantum dots and the intensity decays monotonically with increasing angle. In contrast, the $a=420 \mathrm{~nm}$ and $a=500 \mathrm{~nm}$ samples show pronounced photonic effects with strongly reduced emission in a broad stopband centred at $\alpha=0^{\circ}$ and $\alpha=25^{\circ}$, respectively. We compared the measured angle-dependent intensities to a recently developed theory that describes propagation of light that is diffused by ubiquitous disorder present in real photonic crystals ${ }^{21}$. The curves in Fig. 1c are the predictions with no adjustable parameters, using only the measured reflectivity peaks and calculated bandstructure as input. We obtain excellent agreement between experiment and theory, confirming that the emission of the embedded quantum dots is strongly affected by the photonic crystal.

The decay rate of the excited state of the quantum dots in the photonic crystal was measured by exciting with a short optical pulse and recording the spontaneous emission as a function of time. In photonic crystals, the LDOS and (as a result) the decay rate ideally vanish over a frequency range known as the photonic bandgap. In real crystals with finite dimensions, the LDOS is never zero, but strongly modulated pseudogaps are anticipated ${ }^{19,20}$. In such gaps, vacuum fluctuations are expelled from the crystal, and the spontaneous decay of an excited emitter is inhibited. Away from a gap, the LDOS is increased and spontaneous emission will be enhanced. Figure 2 shows decay curves measured on three different photonic crystals at selected optical frequencies. Two main contributions are present in the data: a fast decay ( $\sim 1 \mathrm{~ns})$ and a slow decay ( $>10 \mathrm{~ns})$. The fast decay is caused by defect-related luminescence of the titania backbone, as confirmed from measurements on bare titania samples. The slow decay is due to the quantum dots, and is easily distinguished from the titania emission owing to its markedly different decay time. Visual inspection of the decay curves in Fig. 2 reveals both accelerated and inhibited decay rates compared to that measured in the non-photonic sample $(a=370 \mathrm{~nm})$. We obtain lifetimes of $9.6 \pm 0.1 \mathrm{~ns}(a=420 \mathrm{~nm})$ and $19.3 \pm 0.2 \mathrm{~ns}$ $(a=500 \mathrm{~nm})$ as compared to a reference value of $12.4 \pm 0.1 \mathrm{~ns}$ $(a=370 \mathrm{~nm})$.

The decay curves of the quantum dots are not single exponentials, which was revealed in additional measurements over longer decay times. Non-single exponential decays in the photonic crystal might be related to fractional decay ${ }^{13,14}$ or a distribution of quantum dots over sites with different LDOS. However, it was recently concluded that fluctuations in the environment surrounding the quantum dots induce a broad distribution of non-radiative decay channels,

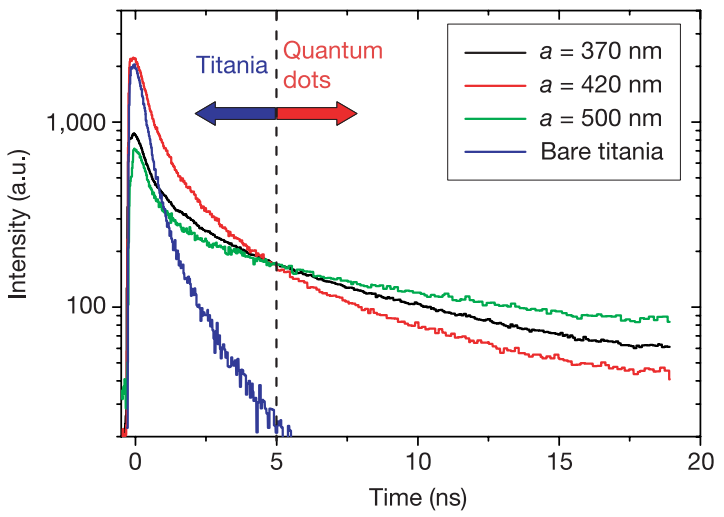

Figure 2 Luminescence decay curves of quantum dots inside three different photonic crystals. The data are recorded at frequencies $15,670 \mathrm{~cm}^{-1}$ ( $a=370 \mathrm{~nm}$, black curve) and $15,100 \mathrm{~cm}^{-1}(a=420 \mathrm{~nm}$, red curve, and $a=500 \mathrm{~nm}$, green curve). The curves have been overlapped after $5 \mathrm{~ns}$. The first part of the decay curve is influenced by emission of titania (blue curve, recorded at $15,400 \mathrm{~cm}^{-1}$ ). After $5 \mathrm{~ns}$ this contribution is negligible. a.u., arbitrary units. explaining the non-single exponential decays and effectively reducing the quantum efficiency ${ }^{22}$. We find that this distribution is centred on the radiative lifetime, and has a wide asymmetric tail of lifetimes from non-radiative contributions that dominates over asymmetries induced by the photonic crystal. Consequently, we consider only photonic effects on the averaged lifetime that is obtained by investigating the quantum-dot decay curve up to $\sim 20$ ns, which is least sensitive to background counts and can be well approximated by a single exponential. The robustness of this method and the sensitivity to variations in the radiative decay rate were carefully checked by comparing to a fit of the decay curve based on the distribution of lifetimes. The observed lifetimes in the inverse opals are all systematically shorter than the lifetime of quantum dots in solution, which is attributed to the reduced quantum efficiency. The reduction in quantum efficiency limits the amount of enhancement and inhibition observed. Nonetheless, the data in Fig. 2 demonstrate a strong variation in the lifetime by a factor of two for photonic crystals with different lattice parameters. These measurements are the first experimental demonstration of the use of three-dimensional photonic crystals to control the radiative lifetime of emitters.

A detailed study of the frequency dependence of the total decay rate has been carried out for crystals with many different lattice parameters, four of which are shown in Fig. 3. The total decay rate is the sum of the radiative and non-radiative decay rates. For each sample, we observe a variation of the total decay rate with emission frequency of more than $50 \%$, which indicates that the radiative decay strongly depends on the emission frequency. For comparison, the dotted orange curve in Fig. 3 is the calculated radiative decay rate for an electric dipole in a homogeneous medium, which scales with the cube of the emission frequency ${ }^{10,11}$. We obtain excellent agreement with this simple frequency scaling on the non-photonic reference sample $(a=370 \mathrm{~nm})$. A more sophisticated model for the emission of an ensemble of quantum dots would require knowledge of the size dependence of the transition-dipole moment, homogeneous broadening, and non-radiative contributions to the decay rate, and is beyond the scope of this Letter. Importantly, the nonradiative contributions are identical for each of the samples, as quantum dots from the same preparation batch were used and all photonic crystals were fabricated under identical conditions. Therefore, variations between samples with different lattice parameters are due to changes in the radiative lifetime.

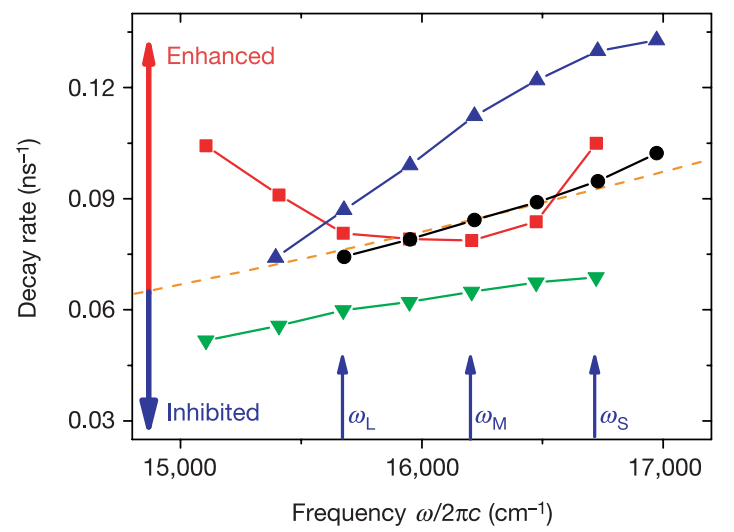

Figure 3 Measured decay rates of the excited states of quantum dots in photonic crystals with different lattice parameters. Lattice parameters are $a=370 \mathrm{~nm}$ (black circles),

$a=420 \mathrm{~nm}$ (red squares), $a=500 \mathrm{~nm}$ (green upside-down triangles) and $a=580 \mathrm{~nm}$ (blue triangles). The curves connecting the measurement points are to guide the eye. The dashed curve is the calculated decay rate for a homogeneous medium. Three fixed emission frequencies are specified in the figure, corresponding to large $\omega_{L}$, diameter $6.0 \mathrm{~nm})$, medium ( $\omega_{\mathrm{M}}$, diameter $4.5 \mathrm{~nm}$ ) and small $\left(\omega_{\mathrm{S}}\right.$, diameter $3.8 \mathrm{~nm}$ ) sized quantum dots. 
We observe changes in the lifetime by up to a factor of two at a fixed frequency $\left(\sim 15,100 \mathrm{~cm}^{-1}\right)$. For the photonic crystal with $a=500 \mathrm{~nm}$, a pronounced inhibition of emission of $30 \%$ is observed in a wide bandwidth exceeding $10 \%$. On the sample with $a=420 \mathrm{~nm}$, a strongly enhanced emission is measured, extending the decay rate by more than $40 \%$ relative to the reference value. The decay rates for the $a=580 \mathrm{~nm}$ sample are all enhanced over a broad frequency range. These measurements demonstrate that photonic crystals offer an effective way of controlling the radiative lifetime through the lattice parameter $a$.

To compare experimental data on different samples with theory, it is instructive to scale the emission frequency with the lattice parameter $(a)$ and the speed of light in vacuum $(c)$ according to $\tilde{\omega}=\omega a / 2 \pi c$. At fixed emission frequency $\omega$, we compare quantum

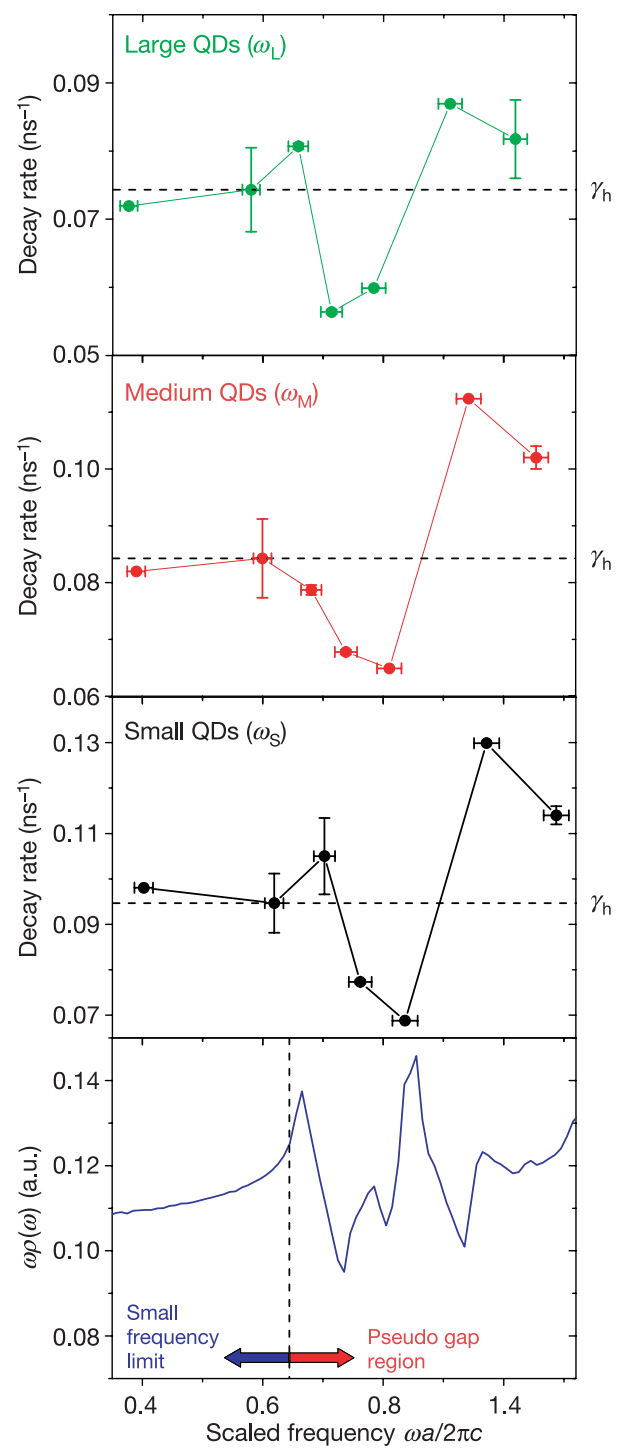

Figure 4 Measured decay rate at three fixed frequencies, $\omega_{L}, \omega_{M}$ and $\omega_{S}$, corresponding to large, medium and small sized quantum dots, when varying the lattice parameter $a$. The plotted curve (bottom) is the emission frequency $\omega$ multiplied by the calculated DOS $\rho(\omega)$ for a titania inverse opal, which is proportional to the decay rate according to Fermi's golden rule. Also indicated (dashed horizontal lines) are the decay rates $\gamma_{\mathrm{h}}$ in the limit of a homogeneous medium. The error bars indicate the standard error of the measurement. The vertical error bars are obtained by averaging over different samples with the same lattice parameters, and by probing different parts of the same sample, while the horizontal error bars are from measurements of the lattice parameter with a scanning electron microscope. dots of the same size and hence with identical emission properties. Figure 4 displays the measured total decay rate as a function of scaled frequency for three fixed emission frequencies corresponding to large $\left(\omega_{L}\right)$, medium $\left(\omega_{M}\right)$ and small $\left(\omega_{S}\right)$ sized quantum dots. The experimental data are compared to the calculated density of states (DOS). The DOS is the unit-cell averaged LDOS weighted by all Bloch mode functions, and approximates the LDOS well in the weakly photonic limit $\tilde{\omega} \ll 1$, where spatial variations are less pronounced. We compare the data to the DOS because it can readily be calculated, whereas LDOS calculations are a tremendous computational task ${ }^{20}$ and existing simulations are currently under debate $^{23}$. At low scaled frequencies $(\tilde{\omega}<0.65)$, the photonic crystal is effectively a homogeneous medium, and we measure a nonphotonic reference decay rate $\gamma_{\mathrm{h}}$. As $a$ is increased, the decay rate is reduced for all quantum dot sizes, and a broad band of inhibited emission is observed in agreement with previous continuous-wave measurements ${ }^{24}$. The inhibition is found in the pseudogap region where the DOS is strongly altered. The rapid drop in the measured decay rate is qualitatively described by the DOS. At even higher frequencies $(\tilde{\omega}>0.85)$, the decay rates are strongly enhanced compared to the reference decay rate over a wide frequency range. In this range, the calculated DOS also reveals enhancements, but this is the strongly photonic regime where the DOS is no longer an adequate description, and local variations described by the LDOS are crucial.

In most previous studies on emission in photonic crystals, dye molecules have been used as light sources. Recent calculations confirm that earlier claims of lifetime effects were not caused by the photonic structure ${ }^{25}$. A fivefold inhibition of the radiative decay rate was concluded from a continuous-wave measurement of the integrated emission performed with a very low quantum efficiency light source ${ }^{24}$. In contrast, a photonic effect on the emission lifetime is complementary and requires high quantum efficiency of the light source. Direct comparison between the present time-resolved and previous continuous-wave experiments is not warranted. In the present measurements, the complex decay curves imply that an averaged lifetime is derived. Interpretation of long-time components of the decay curves in terms of local variations in the lifetime is restricted by fluctuations in the non-radiative decay rate $^{22}$. Furthermore, the non-radiative decay reduces the quantum efficiency. Therefore, a less pronounced modification of the total decay rate is observed in our time-resolved experiment, consistent with the continuous-wave experiment that provides an upper bound. Our lifetime measurements are distinctive because they provide actual control of the population of the excited states, which was not achieved previously.

Our findings of the modified lifetime in a photonic crystal can be compared to measurements of the Purcell effect in high-finesse cavities. Strong enhancement by up to a factor of five $e^{26,27}$ and vacuum-Rabi oscillations ${ }^{28}$ have been observed on resonance in cavity quantum electrodynamic experiments. However, this enhancement is limited to a narrow bandwidth of $\Delta \omega / \omega \approx 10^{-4}$, determined by the linewidth of the cavity. Inhibition of spontaneous emission by a factor of ten in a bandwidth of $10^{-3}$ has been measured off-resonance in sidewall-coated microcavities ${ }^{27}$. Our measurements of both inhibition and enhancement using photonic crystals extend over an unprecedentedly wide bandwidth of more than $10 \%$, which is 100 times wider than in cavities. Furthermore, a serious restriction in a cavity is that control is limited to the small volume of a cavity (typically of the order of wavelength cubed), whereas in the current experiment the quantum dots were located throughout the large volume of the whole photonic crystal $\left(\sim 1 \mathrm{~mm}^{3}\right)$. This clearly demonstrates the advantage of photonic crystals for broadband and large-volume applications with potential for efficient light sources and photon harvesting. We expect our results to open novel avenues for controlling quantum coherence in condensed matter environments ${ }^{18}$. 


\section{Methods}

\section{Sample preparation}

The experiment was performed with titania inverse opals that consist of a face-centred cubic (f.c.c.) structure of air spheres in a titania backbone material. The inverse opals were fabricated by inverting template assisted self-assembled structures ${ }^{16}$. Typical dimensions of the samples were $2 \times 2 \times 0.3 \mathrm{~mm}^{3}$. The high quality of the crystals is quantified by the scattering mean free path of light propagation, which was $15-20 \mu \mathrm{m}$. In total, ten different samples were studied with lattice parameters ranging from $a=240 \mathrm{~nm}$ to $650 \mathrm{~nm}$. The photonic crystals were infiltrated for $24 \mathrm{~h}$ with a mixture of $50 \%$ chloroform and $50 \%$ butanol containing $10^{-7} \mathrm{~mol} \mathrm{litre}{ }^{-1} \mathrm{ZnSe}$-coated CdSe colloidal quantum $\operatorname{dots}^{29}$. Afterwards the samples were rinsed for one minute in chloroform and dried. The quantum dots had a size dispersity of $5 \%$ around an average diameter of $4.5 \mathrm{~nm}$. The infiltration led to a distribution of quantum dots on the surfaces of the air spheres inside the inverse opal with a concentration of less than 10 quantum dots per air sphere. This is sufficiently low to avoid energy transfer between quantum dots. The ensemble of quantum dots in the solution had a lifetime of around $20 \mathrm{~ns}$ and a quantum efficiency of $50 \% \pm 5 \%$; the latter was measured by comparing absorption and emission to a R6G dye standard. To minimize oxidation and contamination, all sample preparation and handling was carried out in a nitrogen-purged glove box, and the optical measurements were performed in a sealed chamber under 1.7 mbar nitrogen.

\section{Experimental set-up}

Optical excitation of the quantum dots was performed with either a continuous-wave $\operatorname{argon}$ laser $(488 \mathrm{~nm})$ or a pulsed diode laser $(100 \mathrm{ps}, 440 \mathrm{~nm})$. The former was used for the spectral measurements and the latter for time-resolved experiments. The excitation beam was coupled through a single-mode fibre and illuminated the photonic crystal. The fibre and the sample were mounted on the same rotational stage, which allowed changing of the detection angle while keeping the excitation volume constant. The spontaneously emitted light was detected from the surface facing away from the excitation beam at an angle $\alpha$ relative to the (111) planes of the f.c.c. lattice. The light was collected in a cone of $\sim 8^{\circ}$ around the detection angle, dispersed with a spectrometer $(2 \mathrm{~nm}$ resolution), and detected with a fast photomultiplier. Time-correlated single-photon counting was implemented to measure the arrival time of the emitted photons. Careful measurements on reference samples ruled out the presence of energy transfer ${ }^{30}$, reabsorption, and other non-photonic effects on the lifetime.

\section{Data analysis}

The calculated curves in Fig. 1c were obtained with only experimental data from separate reflectivity experiments as input to the theory, combined with band structure calculations ${ }^{21}$. Furthermore, the lattice parameters of the photonic crystals were taken from scanning electron microscope measurements.

The decay curves in Fig. 2 were obtained by binning the arrival times of the single photons detected with the photomultiplier. Contributions from dark counts in the photomultiplier were measured and subtracted from the data. The first $5 \mathrm{~ns}$ were discarded from each decay curve, as defect-related luminescence of titania was contributing in this range. The signal beyond $5 \mathrm{~ns}$ was unaffected by titania luminescence, and the decay until $\sim 20 \mathrm{~ns}$ was modelled with a single exponential decay from which the lifetime was extracted. Whereas strong lifetime effects were observed on the efficient quantum dots, we observe no systematic change of the lifetime from the low quantum efficiency titania luminescence.

The calculated lifetime of quantum dot emission in a homogeneous medium in Fig. 3 is given by $\tau_{\mathrm{h}}=C / \omega^{3}$. Here $C=3 \hbar \varepsilon_{0} \pi c^{3} / n \bar{d}^{2}$ contains Planck's constant $\hbar$, the vacuum permittivity $\varepsilon_{0}$, the speed of light in vacuum $c$, the refractive index $n$, and the mean transition-dipole moment $\bar{d}$ averaged over the orientation and size distribution of the quantum dots. We assume an average refractive index of $n=1.17$ and arrive at a mean dipole moment of 3.0 atomic units.

Received 25 February; accepted 16 June 2004; doi:10.1038/nature02772.

1. Yablonovitch, E. Inhibited spontaneous emission in solid-state physics and electronics. Phys. Rev. Lett 58, 2059-2062 (1987)

2. Painter, O. et al. Two-dimensional photonic band-gap defect mode laser. Science 284, 1819-1821 (1999).

3. Smith, C. J. M. et al. Near-infrared microcavities confined by two-dimensional photonic bandgap crystals. Electron. Lett. 35, 228-230 (1999).

4. Colombelli, R. et al. Quantum cascade surface-emitting photonic crystal laser. Science 302, 1374-1377 (2003).

Noda, S. et al. Polarization mode control of two-dimensional photonic crystal laser by unit cell structure design. Science 293, 1123-1125 (2001).

6. Kim, J., Benson, O., Kan, H. \& Yamamoto, Y. A single-photon turnstile device. Nature 397, 500-503 (1999).

7. Michler, P. et al. A quantum dot single-photon turnstile device. Science 290, 2282-2285 (2000).

8. Kuhn, A., Hennrich, M. \& Rempe, G. Deterministic single-photon source for distributed quantum networking. Phys. Rev. Lett. 89, 067901 (2002).

9. Grätzel, M. Photoelectrochemical cells. Nature 414, 338-344 (2001).

10. Scully, M. O. \& Zubairy, M. S. Quantum Optics (Cambridge Univ. Press, Cambridge, 1997).

11. Loudon, R. The Quantum Theory of Light (Oxford Univ. Press, New York, 2000).

12. Bykov, V. P. Spontaneous emission from a medium with a band spectrum. Sov. J. Quant. Electron. 4 861-871 (1975).

13. Lambropoulos, P., Nikolopoulos, G. M., Nielsen, T. R. \& Bay, S. Fundamental quantum optics in structured reservoirs. Rep. Prog. Phys. 63, 455-503 (2000).

14. John, S. \& Quang, T. Spontaneous emission near the edge of a photonic band gap. Phys. Rev. A $\mathbf{5 0}$ 1764-1769 (1994)

15. John, S. Strong localization of photons in certain disordered dielectric superlattices. Phys. Rev. Lett. 58, 2486-2489 (1987)
16. Wijnhoven, J. E. G. J. \& Vos, W. L. Preparation of photonic crystals made of air spheres in titania. Science 281, 802-804 (1998)

17. Vahala, K. J. Optical microcavities. Nature 424, 839-846 (2003).

18. Mabuchi, H. \& Doherty, A. C. Cavity quantum electrodynamics: coherence in context. Science 298, 1372-1377 (2002).

19. Sprik, R., van Tiggelen, B. A. \& Lagendijk, A. Optical emission in periodic dielectrics. Europhys. Lett. 35, 265-270 (1996).

20. Busch, K. \& John, S. Photonic band gap formation in certain self-organizing systems. Phys. Rev. E 58, 3896-3908 (1998).

21. Koenderink, A. F. \& Vos, W. L. Light exiting from real photonic band gap crystals is diffuse and strongly directional. Phys. Rev. Lett. 91, 213902 (2003).

22. Fisher, B. R., Eisler, H.-J., Stott, N. E. \& Bawendi, M. G. Emission intensity dependence and singleexponential behavior in single colloidal quantum dot fluorescence lifetimes. J. Phys. Chem. B 108, 143-148 (2004).

23. Wang, R., Wang, X.-H., Gu, B.-Y. \& Yang, G.-Z. Local density of states in three-dimensional photonic crystals: Calculation and enhancement effects. Phys. Rev. B 67, 155114 (2003)

24. Koenderink, A. F., Bechger, L., Schriemer, H. P., Lagendijk, A. \& Vos, W. L. Broadband fivefold reduction of vacuum fluctuations probed by dye in photonic crystals. Phys. Rev. Lett. 88, 143903 (2002).

25. Li, Z. Y. \& Zhang, Z. Q. Weak photonic band gap effect on the fluorescence lifetime in threedimensional colloidal photonic crystals. Phys. Rev. B 63, 125106 (2001).

26. Gérard, J. M. et al. Enhanced spontaneous emission by quantum boxes in a monolithic optical microcavity. Phys. Rev. Lett. 81, 1110-1113 (1998).

27. Bayer, M. et al. Inhibition and enhancement of the spontaneous emission of quantum dots in structured microresonators. Phys. Rev. Lett. 86, 3168-3171 (2001).

28. Hood, C. J., Chapman, M. S., Lynn, T. W. \& Kimble, H. J. Real-time cavity QED with single atoms. Phys. Rev. Lett. 80, 4157-4160 (1998).

29. Dabbousi, B. O. et al. (CdSe) ZnS core-shell quantum dots: synthesis and characterization of highly luminescent nanocrystallites. J. Phys. Chem. B 101, 9463-9475 (1997)

30. Crooker, S. A., Hollingsworth, J. A., Tretiak, S. \& Klimov, V. I. Spectrally resolved dynamics of energy transfer in quantum-dot assemblies: towards engineered energy flows in artificial materials. Phys. Rev. Lett. 89, 186802 (2002).

Acknowledgements We thank L. Woldering for sample preparation, F. Koenderink for DOS calculations, A. Mosk and J. Kelly for discussions, and A. Lagendijk for support. This work is part of the research programme of the Stichting voor Fundamenteel Onderzoek der Materie (FOM), which is financially supported by the Nederlandse Organisatie voor Wetenschappelijk Onderzoek (NWO).

Competing interests statement The authors declare that they have no competing financial interests.

Correspondence and requests for materials should be addressed to P.L. (p.lodahl@utwente.nl).

\section{Atomic-scale imaging of nanoengineered oxygen vacancy profiles in $\mathrm{SrTiO}_{3}$}

\section{David A. Muller ${ }^{1,2}$, Naoyuki Nakagawa ${ }^{1,3}$, Akira Ohtomo ${ }^{1,4}$, John L. Grazul ${ }^{1,2}$ \& Harold Y. Hwang ${ }^{1,3,5}$}

${ }^{1}$ Bell Laboratories, Lucent Technologies, Murray Hill, New Jersey 07974, USA ${ }^{2}$ School of Applied and Engineering Physics, Cornell University, Ithaca, New York 14583, USA

${ }^{3}$ Department of Advanced Materials Science, University of Tokyo, Kashiwa, Chiba 277-8561, Japan

${ }^{4}$ Institute of Materials Research, Tohoku University, Sendai, 980-8577, Japan ${ }^{5} J a p a n$ Science and Technology Agency, Kawaguchi, 332-0012, Japan

At the heart of modern oxide chemistry lies the recognition that beneficial (as well as deleterious) materials properties can be obtained by deliberate deviations of oxygen atom occupancy from the ideal stoichiometry ${ }^{1,2}$. Conversely, the capability to control and confine oxygen vacancies will be important to realize the full potential of perovskite ferroelectric materials, varistors and field-effect devices ${ }^{3,4}$. In transition metal oxides, oxygen vacancies are generally electron donors, and in strontium titanate $\left(\mathrm{SrTiO}_{3}\right)$ thin films, oxygen vacancies (unlike impurity dopants) are particularly important because they tend to retain high carrier mobilities, even at high carrier densities ${ }^{5}$. Here we report the successful fabrication, using a pulsed laser deposition technique, of $\mathrm{SrTiO}_{3}$ superlattice films with oxygen doping profiles 